\title{
LABOR-THERAPEUTIC ACTIVITIES AS PART OF THE COMPLEX PHYSIOTHERAPEUTIC AND REHABILITATION PROGRAM AT THE CARPAL CHANNEL SYNDROME
}

\author{
Danelina Vacheva \\ Medical University Hospital - Pleven, Bulgaria
}

\begin{abstract}
Introduction: Carpal tunnel syndrome (CKD) was Compression Syndrome of n. medianus in the carpal canal. Functional violations are observed of grip (precise top grip, palmar grip, scissor grip, key grip) requiring precise control with thumb opposition. The purpose of the report is a comparative analysis of the results of the two complex physiotherapy and rehabilitation programs in unoperated patients with carpal tunnel syndrome. Material and methods: Observed there were 37 patients with JCC who had completed a complex rehabilitation program involving medication therapy and physiotherapy procedures (paraffin therapy, healing massage, kinesitherapy, electrophoresis with Nivalin, phonophoresis with drug gel, labour-therapeutic). In order to objectify the results, we performed: a comprehensive functional assessment of radial joints, wrist and fingers (Vacheva, 2010) and subjective assessment of the patient for the applied treatment in 5 degrees. Results and Discussion: The results of the applied rehabilitation program show beneficial effects on subjective complaints and objective measurements that require precise control with thumb opposed. Incorporating labour-therapeutic into the rehabilitation program greatly improves fine motor skills and sensitivity, and the entertaining nature of the labour-therapeutic activities influences positively to the emotional state of the patient. Conclusion: Early onset of conservative treatment in patients with mild to moderate CKD symptomatology leads to a possible delay of surgical decompression of $n$. medianus for a later stage.
\end{abstract}

Key words: carpal tunnel, rehabilitation, labor activities.

\section{INTRODUCTION}

The carpal tunnel syndrome (CTS) is described for a first time by Sir James Paget in 1865 as a compression syndrome on the $\mathrm{n}$. medianus in the carpal tunnel (Figure 1) (Ganchev, 1997). The condition

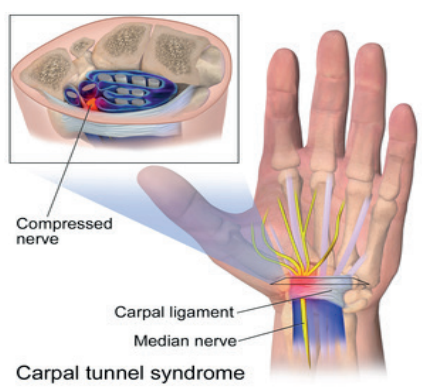

Figure 1. Compression of the n. medianus

Reasons for occurrence of carpal tunnel syndrome are: unspecified inflammation and swelling of flexor tendon and bursae in multiple repeated movements of the same type; hypertrophy of synovial tissue in the carpal tunnel in repetitive movements (work with vibrating equipment); posttraumatic conditions in the area of the wrist joint - soft-tis- results from discrepancy between carpal tunnel volume and its contents (including the median nerve) that leads to expression of neurological symptomatic (motor and sensory disorders) distal from the wrist joint (Figure 2) (Cotta $\mathrm{H}$ et all, 1985).

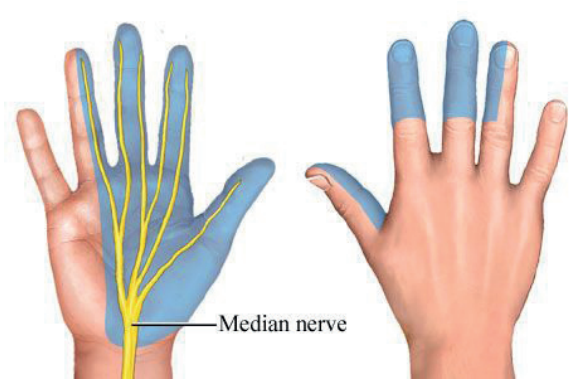

Figure 2. Sensitivity zone of the n. medianus

sue, fracture or carpal luxation; rheumatoid synovitis; last months of pregnancy; systematic diseases (nephropathic amyloidosis) (Vacheva, 2015); patients on hemodialysis; tumor formations; ganglion or lipoma localized in the carpal tunnel (Boychev, 1963). 
The clinical symptoms (Gerberman et al., 1984) are expressed as pain (predominantly during the night) and paresthesia in I-III finger, spreading out to the forearm; heavily changed or lack of tactile gnosis; morning stiffness and decreased mobility of the wrist joint and metacarpophalangeal joints of fingers (thumb, II and III finger); increased intensity of pain in the wrist joint and fingers in repetitive movements; other sensory and motoric disorders at the lower branch of $n$. medianus. In cases of prolonged complaints weakness and atrophy of thenar

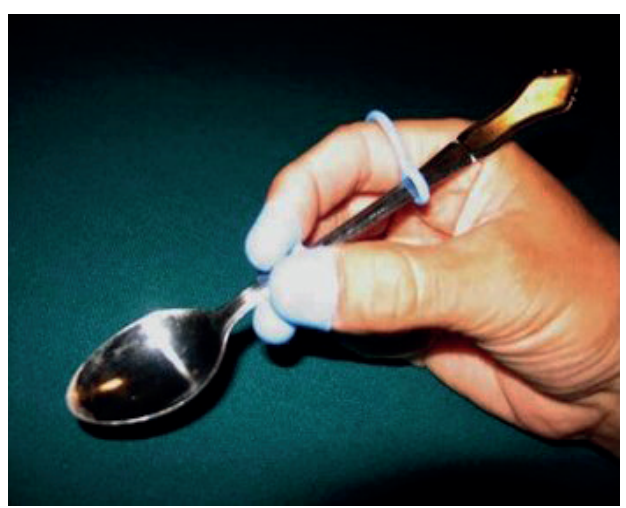

Figure 3. Activities requiring performance of palmar grip

Object of the communication is a comparative analysis of results from conducted comprehensive physiotherapy and rehabilitation program (with and without labor therapy) for patients with carpal tunnel syndrome who have not undergone surgery.

\section{METHODOLOGY}

The Clinic of Physical and Rehabilitation Medicine at the University Hospital of Pleven has a long-term experience in treating patients who suffer traumas and arm diseases. In 2009 in the comprehensive rehabilitation program a labor therapeutic procedure was included (after opening an office for labor therapy) to support rehabilitation of arm and stimulate the functional recovery of the limb (Vacheva, 2018). For the period January 2018 - May 2019, 37 patients with electromyographically diagnosed symptomatic of carpal tunnel syndrome (lightly to moderately expressed) asked for physiotherapeutic help in the Clinic of Physical and Rehabilitation Medicine at the University Hospital of Pleven. Two (three) rehabilitation courses of 7 day duration are conducted (under clinical pathway or as outpatients). Patients' average age is 45,6 (from 25 to 57 years old). In 21 patients the dominant limb is affected $(56,8 \%)$, in $12(32,4 \%)$ - the non-dominant limb, and in $4(10,8 \%)$ - both limbs. The prolongation of the complaints before physiotherapy and rehabilitation treatment is 1 to 3 months (1,9 months muscles are observed, especially of abductor pollicis brevis and $\mathrm{m}$. opponens pollicis (Bosnev, Matev., 1989).

Functional disorders are also observed when gripping actions are performed (precise pinch grip, palm grip - Fig. 3, side-to-side grip, pad-to-side grip) that require precise control through opposition of the thumb (Vacheva, 2017), as well as difficulty or complete impossibility to perform repetitive movements in the wrist joint and fingers (Bosnev, 1998).

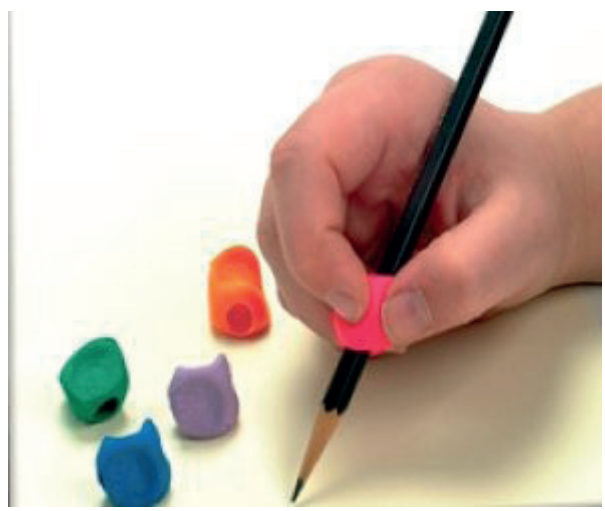

on average).

In order to objectivize the results, the patients are examined using the following methods, described in an individual rehabilitation record (Matev, Bankov, 1977):

$\checkmark$ Somatic status - weakness and hypotrophy of thenar muscles, increased pain in repetitive movements ;

$\checkmark$ Neurological status - decreased or lack of sensuousness in n. medianus autonomic area;

$\checkmark$ Kinesiological status - limited scope of movement in wrist joints and metacarpophalangeal joints of thumb, II and III finger;

- Functional status - complete functional assessment test of radioulnar joints, wrist joints and fingers (CFA by Vacheva, 2010), covering Visual Analogue Scale of Pain (VAS), goniometry, Manual Muscle Test (Bankov, et.al, 1987), grips, Everyday Life Activities Test (ADL);

$\checkmark$ Patient's subjective assessment of the effectiveness of applied therapy, using a 5-level scale: level 1 - intensified complaints; level 2 - no improvement; level 3 - with improvement; level 4 - considerable improvement; level 5 vast improvement;

$\checkmark$ Instrumental methods - electromyography examinations (EMG) to diagnose light to 
T-test of Student and Wilcoxon rank test is performed during the statistical processing of results.

The comprehensive rehabilitation program includes (Gatev, et al, 1992):

- Medication therapy (Nonsteroidal anti-inflammatory drugs and vitamins from the B groups);

- Physiotherapic procedures:

$\checkmark$ Therapeutic massage for improving the function of forearm and arm's affected muscle;

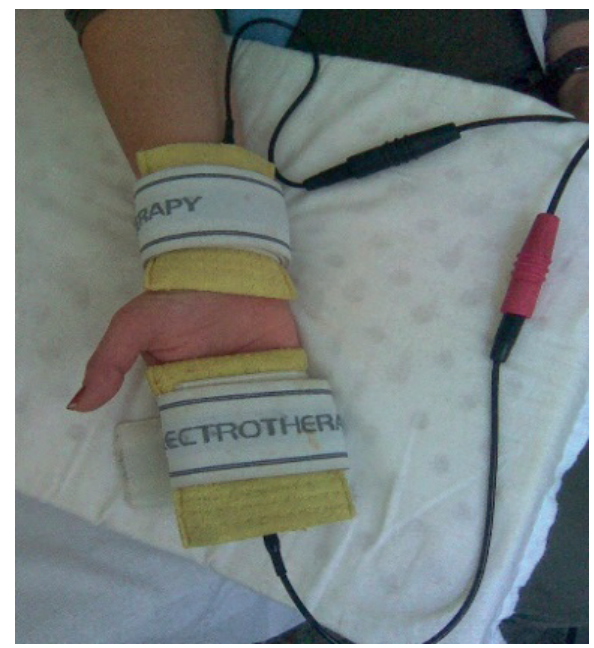

Figure 4. Nivalin electrophoresi

Labor therapy for improving precise grips (Bankov, et. al, 1979), arm's coordination and sensuousness (Paskaleva, 2017): working with paper, cardboard and wooden material;
Kinesiotherapy with mobilization techniques and analytic gymnastics that includes active exercised for strengthening muscles of forearm and fingers (Karaneshev, 1983);

$\checkmark$ Electrophoresis with nivalin (Figure 4 - longitudinal method; $15-20 \mathrm{~min}$. according to individual's sensitivity);

$\checkmark$ Phonophoresis with nonsteroidal anti-inflammatory drugs (gel) $0,6-0,8 \mathrm{~W} / \mathrm{cm}^{2}, 8-10 \mathrm{~min}$., labile method (Figure 5);

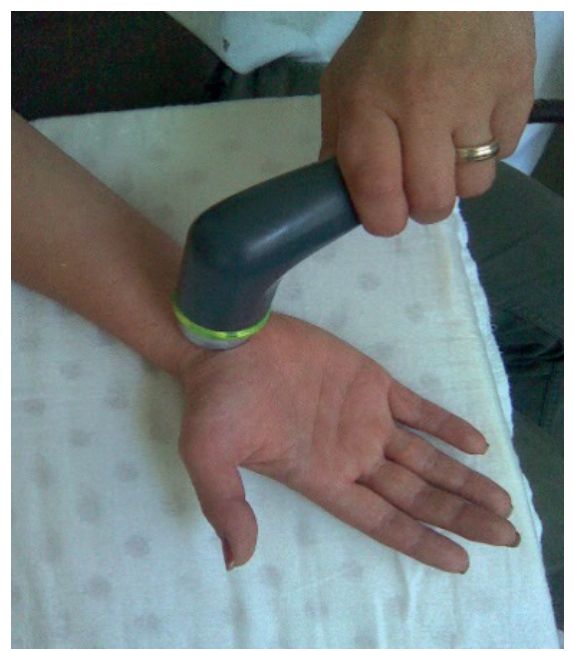

Figure 5. Phonophoresis with NAID (gel)

working with yarn and textile; working with plastics (Karaneshev, 1982; Kapteli, Lasskaya, 1979) (Figure 6).
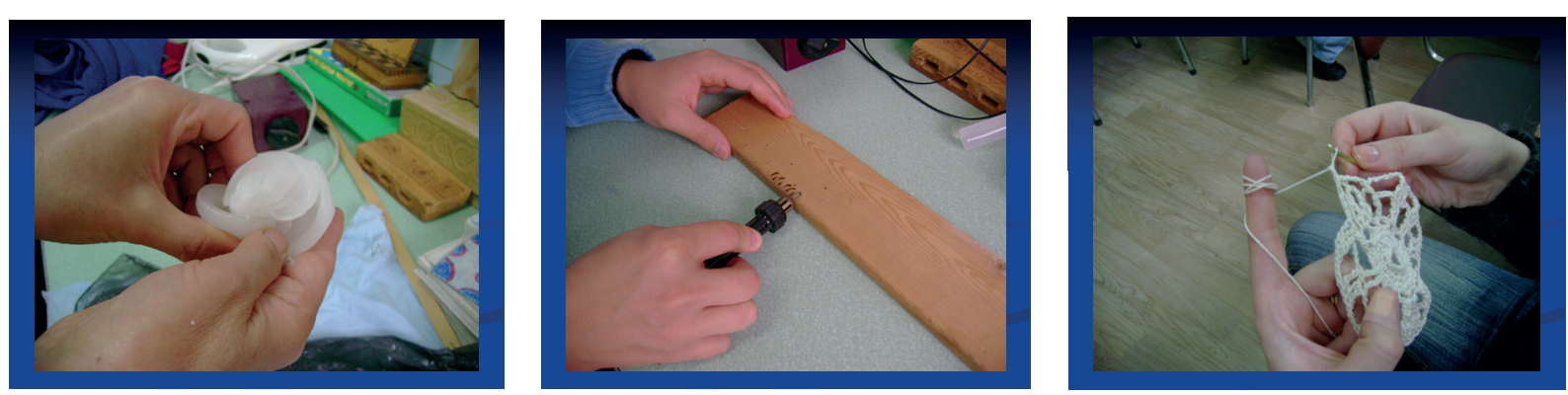

Figure 6. Occupational therapy activities

\section{RESULTS AND DICUSSION}

Achieved results are compared to results of patients, who had rehabilitation before 2009 , when the rehabilitation program did not include labor therapy, representing a control group (CG): 49 patients, 27 of which had damaged dominant limb $(55,1 \%)$, 15 - non-dominant limb (30,6\%) and 7 - damaged both limbs (14,3\%). The Clinic maintains a database with patients who underwent treatment. Figure 7 shows that the allocation of patients according to damaged limbs has no statistical differences $(\mathrm{KW}=44$, a $\mathrm{P}>0,005)$ and reasoning and conclusions can be made. 
control group

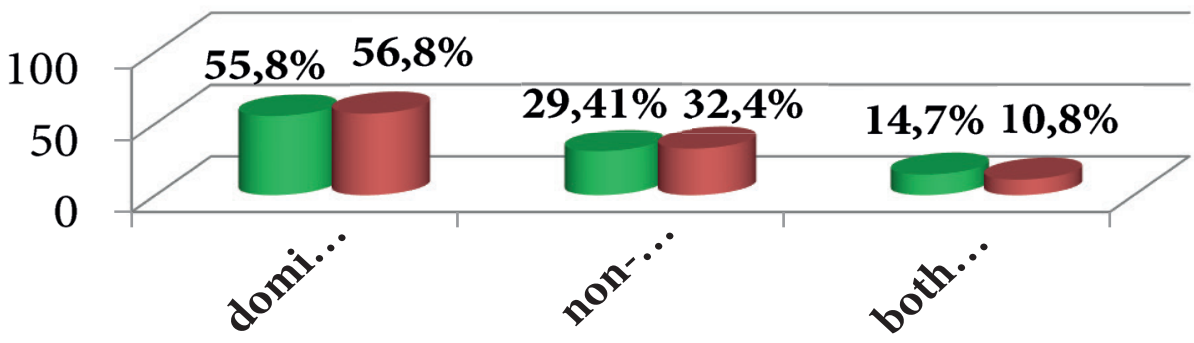

Figure 7. Distribution of patients by dominance of the injured limb

For the purpose of the current scientific commu- the Everyday Life Activities Test where improved nication the statistical processing of results from results in functional recovery of patients rehabiliEveryday Life Activities Test and Complete Fun- tated through comprehensive physiotherapy with ctional Assessment Test on forearm, wrist joint and included labor therapy is demonstrated ( $F=24,58$, fingers is presented. Figure 8 shows the results from $\mathrm{P}<0,0001 ; \mathrm{KW}=66,2$ at $\mathrm{P}=0,0001$ ).

\section{5

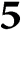 \\ 4}
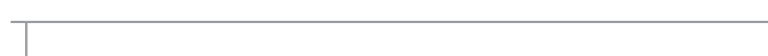

2

1

$\mathbf{0}$

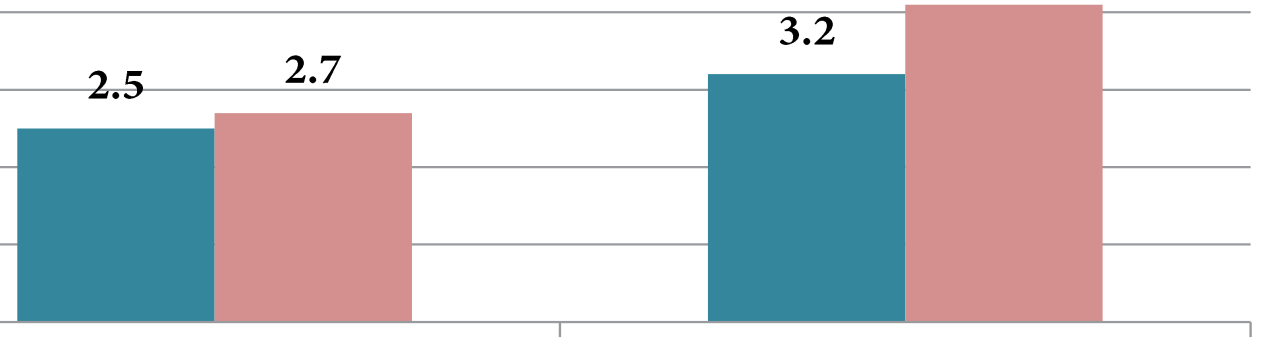

before rehabilitation

Figure 8. Level of self-care (household and work activities) of patients with Control Group and Experimental Group before and after rehabilitation

Essential for reporting functional recovery of upper limb is the option to perform various domestic and labor activities related to the everyday life, where the initial status of the damaged limb and its improvement level are of significance. Such data is presented in Table 1 and prove better values in patients from the experimental group (EG) in comparison to the patients who had rehabilitation without labor activities.

Table 1. Differences between the end and the beginning of the rehabilitation process for the two groups of the ADL (household and work activities) test

\begin{tabular}{|c|c|c|c|c|c|c|c|c|}
\hline \multicolumn{4}{|c|}{ Control group } & \multicolumn{4}{c|}{ Experimental group } & \multirow{2}{*}{ P } \\
\cline { 2 - 7 } & $\mathrm{X}$ & $\mathrm{S}$ & $\begin{array}{l}\text { Coeff. } \\
\text { of var. }\end{array}$ & $\mathrm{n}$ & $\mathrm{X}$ & $\mathrm{S}$ & $\begin{array}{c}\text { Coeff. } \\
\text { of var. }\end{array}$ & \\
\hline 49 & 0,64 & 0,17 & 24,63 & 37 & 1,11 & 0,24 & 21,82 & $<0,05$ \\
\hline
\end{tabular}

Figure 9 represents the results for patients from 17\% of all patients are in „satisfactory” functional the Complete Functional Assessment Test, in the beginning and at the end of the rehabilitation procondition; between the compared groups no sigof cess, and it can be seen that at the beginning of of the therapy the patients form the experimental the rehabilitation most of the patients are within the limits of "good" functional status and only group show better results and $81,8 \%$ of them have recovered to the level of „very good” and "excel- 
lent" functional status; the patients from the con- have not been influenced positively by the consertrol group are 51,02\%. Both groups contain patients vative treatment and remain in the limits of "satis(control group -3 , experimental group - 2) who factory" recovery $(\mathrm{P}<0,05)$.

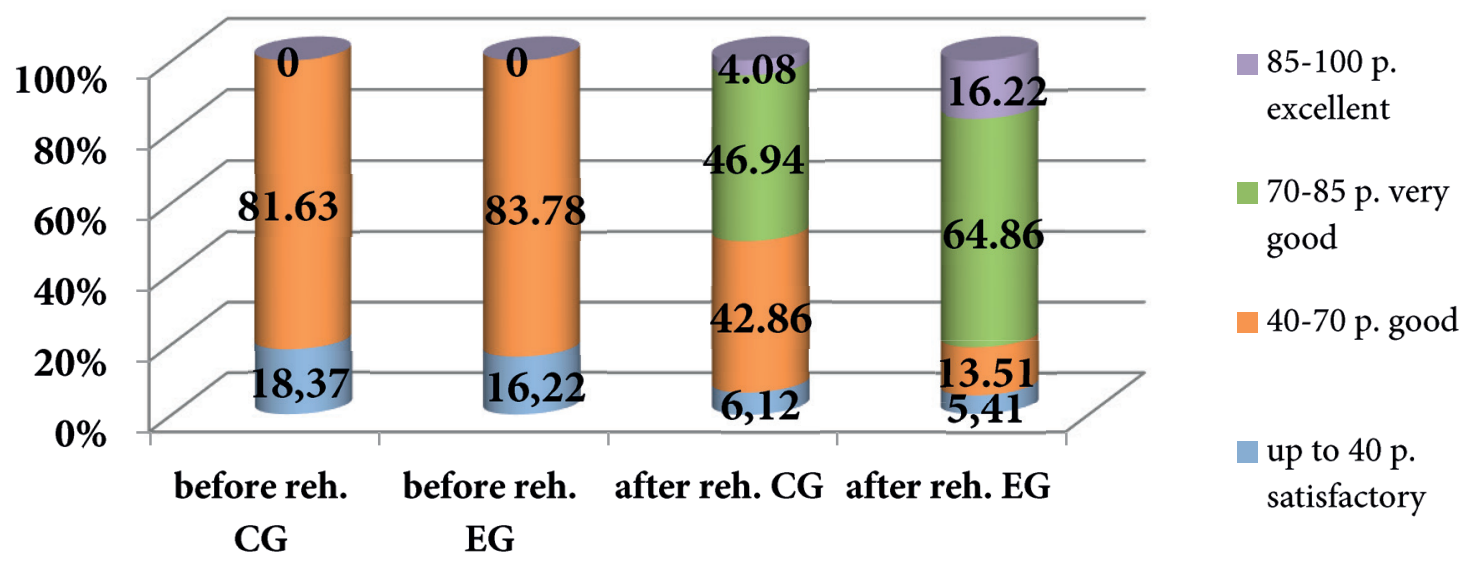

Figure 9. The degree of complex functional status of the patients at the beginning and end of the rehabilitation process

\section{Reasoning}

1. The results from the applied physiotherapeutic program show statistically credible favorable influence $(\mathrm{p}<0,001)$ on subjective complaints (pain), reduced mobility (grip) and functional disorders (Everyday Life Activities) that require precise control through opposition of the thumb.

2. Inclusion of labor and domestic activities form the everyday life in the comprehensive physiotherapy program significantly improves fine motor and sensuousness.

3. The recreational character of the labor therapeutic activities influences positively the emotional status of the patients.

\section{CONCLUSION}

Timely initiation of conservative treatment with medications and the presented comprehensive rehabilitation program (with labor therapy) in patients with light to moderately expressed symptomatic of carpal tunnel syndrome leads to favorable influence on subjective and objective symptoms and to a better functional recovery, and the purpose is deferred surgical decompression of $\mathrm{n}$. medianus to a later stage.

\section{REFERENCES}

Bankov, St. et al. (1979). Occupational therapy, Medicine and physical culture, Sofia, pp. 7-25; 117-130; 136-143 [In Bulgarian]. Bankov, St., Krasteva, V., Vajarov, Q. (1987). Manual muscular testing with the basics of kinesiology and ptokinesiology, Medicine and physical culture, Sofia, pp. 198-210 [In Bulgarian].

Bosnev, V. (1998). Neurovegetative hand pathology, Medicine and physical culture, Sofia, pp. 136-142 [In Bulgarian].

Bosnev, V., Matev, I. (1989). Diseases of the hand, Medicine and physical culture, Sofia, pp. 174-175, 230240 [In Bulgarian].

Boychev, B. (1963). Orthopedics and traumatology, Medicine and physical culture, Sofia, pp. 251-253 [In Bulgarian].

Cotta, H., Heipertz, W., Kersten, H. (1985). Orthopedie, Georg Thieme Vergal, Stuttgart - New York, pp. 231247.

Ganchev, M. (1997). Clinical orthopedics, K\&M, Sofia, pp. 278-281 [In Bulgarian].

Gatev, St., Bankov, St., Busarov, St. (1992). Physical Therapy Guide, Medicine and physical culture, Volume 2, Sofia, pp. 193-194 [In Bulgarian].

Gerberman, R H. et al. (1984). Carpal tunnel pressures and wrist position in patients with Colles-fractures. Trauma, (24), pp. 747.

Kaptelin, LF., Lasskaia, LA. (1979). Occupational therapy in traumatology and orthopedics, Medicine, Moscow, pp. 101-132 [In Russian].

Karaneshev, G. (1982). Functional occupational therapy, Second edition, National Sport Academy Pres, Sofia, pp. 5-56 [In Bulgarian].

Karaneshev, G., Sokolov, B., Venova, L. et al. Edited by G. Karaneshev. (1983). Theoty ang methods of remedial gymnastics, Medicine and physical culture, Sofia, pp. 
188-201 [In Bulgarian].

Matev, I., Bankov, St. (1977). Rehabilitation of lesions of the hand, Medicine and physical culture, Sofia, pp. 94-95 [In Bulgarian].

Vacheva, D. (2018). Development of Activity of LabourAnd Occupational Therapies in University Hospital, Pleven, Bulgaria. Biomed J Sci \& Tech Res, vol. 11 (1), pp. 1-3.

Vacheva, D. (2015). Medical Rehabilitation and Occupational Therapy in Patients with Lesion of Plexus Brachialis. AMB, Vol. XLII (1), pp. 56-62.

Vacheva, D. (2017). Rehabilitation Program in Children with Birth Trauma of the Brachial Plexus. GISAP: Medical Science, Pharmacology, March, (12), pp. 3-6.

Paskaleva, R. (2017). Application of occupational therapy and kinesitherapy to improve the driving activity and activities of daily life (ADL) in children with cerebral damage. Medicine \& Science in Sports \& Exercise, Issue 12 (2), Vol. 49, pp. 2875-2884.

\section{Corresponding author:} Danelina Vacheva, PhD Associate Professor Medical University, University Hospital - Pleven, Bulgaria Department "Physical Medicine, Rehabilitation, Ergotherapy and Sports" 5800, Pleven, “G. Kochev” 8a; Bulgaria E-mail: danelina@abv.bg 